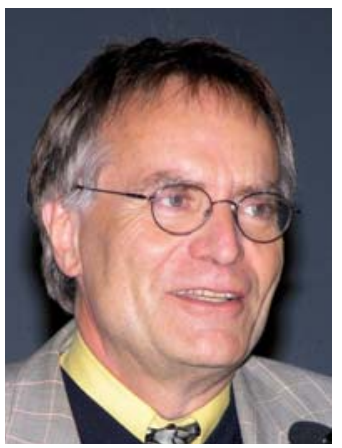

\title{
Weltpremiere: Phytotherapie und Sport
}

Eine Premiere in der Medizin? Am 19. November 2009 ist eine solche geplant: Erstmals beschäftigt sich eine Tagung mit dem Thema Phytotherapie und Sport. Die 1986 gestartete Tradition der Schweizerischen Jahrestagung für Phytotherapie ist damit beim vierundzwanzigsten Thema angelangt: Noch nie hat sich in der Geschichte dieser Veranstaltung eine Thematik wiederholt. Eine Premiere ist nun aber doch ein Novum.

Die Phytotherapie wird als Bestandteil der modernen Medizin immer wieder unterschätzt und zu wenig wahrgenommen. Eine bisher kaum betrachtete Domäne ist die Sportmedizin. Gesundheit und Prophylaxe sind im Sport eine wichtige Komponente. Da ist ein Anknüpfungspunkt zur Phytotherapie, insbesondere im Bereich der Erkältungskrankheiten. Anerkannte Sportärzte berichten von ihrem Umgang mit pflanzlichen Arzneimitteln: Für Wintersportler und Schwimmerlnnen ist der Schutz der Atemwege, die Kälte und Wasser ausgesetzt sind, von höchster Bedeutung. Die Erfahrungen vom Spitzensport in diesem Bereich können umgesetzt werden in den Breitensport und sind deshalb auch für Grundversorger von höchster Bedeutung. Zur Diskussion stehen weitere Themen wie Sportverletzungen, Prävention und Rehabilitation im muskulären Bereich sowie Leistungsförderung und Doping. Erstmals steht auch ein veterinärmedizinischer Workshop auf dem Programm.

Die Schweizerische Medizinische Gesellschaft für Phytotherapie SMGP freut sich als Organisator, mit Simone Niggli-Luder eine Spitzensportlerin unter den Referierenden zu wissen. Auch das ist ein spezielles Angebot. Als Biologin ist sie der Wissenschaft zugetan und kann analysieren, wie und warum sie sich in einer Sportart, die normalerweise jeder Witterung trotzt, ohne Krankheiten und Verletzungen an der Spitze behaupten kann. 15 Goldmedaillen im Orientierungslauf sprechen für sich.

Angestrebt wird traditionsgemäss auch an dieser 24. Schweizerischen Jahrestagung für Phytotherape die Synthese zwischen Wissenschaft und Praxis. Sport spielt in der heutigen Gesellschaft eine grosse Rolle: Er ist ein nicht mehr weg zu denkendes Phänomen. Die einen betreiben keinen Sport, die anderen zuviel. Interessiert ist wohl eine Mehrheit der Bevölkerung. Entsprechend ist Sport in der Medizin ein wichtiges Thema: Wir denken - wieso nicht auch in der Phytotherapie?

Die Volksabstimmung im Mai diesen Jahres hat gezeigt, dass grosse Teile der Bevölkerung der Komplementärmedizin positiv gegenüberstehen. Der Grundversorger sollte diesem gesellschaftlichen Anspruch nicht ausweichen. In der Phytotherapie findet er einen Ansatz, der komplementärmedizinisches Gedankengut kombiniert mit seinen bisherigen Erfahrungen. Die SMGP schafft die Möglichkeit, einzusteigen in ein faszinierendes Teilgebiet moderner Medizin. Sie darf mit Stolz darauf verweisen, dass ihre Fort- und Weiterbildung universitär verankert ist, zeichnen doch jeweils das Institut für Naturheilkunde am UniversitätsSpital in Zürich und die Zürcher Hochschule für angewandte Wissenschaften in Wädenswil für das wissenschaftliche Programm. Dank regelmässigen Evaluationen decken die Veranstaltungen die Bedürfnisse der Teilnehmenden ab. Versuchen Sie den Einstieg - unsere Jahrestagung mit ihrem Ambiente ist eine günstige Gelegenheit.

Prof. Dr. Beat Meier

Geschäftsleitung SMGP / Zürcher Hochschule für angewandte Wissenschaften, Wädenswil

Informationen zur Tagung unter www.smgp.ch.

Direkter Link zum Programm: http://www.smgp.ch/auspro/jtagung/2009/tag09.html

Anmeldungen sind auch kurzfristig möglich. 